\title{
UTILIZAÇÃO DE ÍNDICES $r$ E R OBTIDOS DE PROGRAMAS INTERLABORATORIAIS PARA O CONTROLE DE PRECISÃo DE MÉTODO ANALÍTICO: DETERMINAÇÃo DE ÁGUA POR KARL FISCHER
}

Queenie Siu Hang Chui*

Centro de Ciências Exatas e Tecnológicas, Universidade São Francisco, Rua Alexandre Rodrigues Barbosa, 45, 13251-900 Itatiba - SP

Heloísa Burkhardt Antonoff

Divisão de Química, Instituto de Pesquisas Tecnológicas do Estado de São Paulo S.A., Av. Prof. Almeida Prado, 535, 05508-901

São Paulo - SP

José Carlos Olivieri

Av. Senador Casemiro da Rocha, 1257, ap. 24 bloco A, 04747-003 São Paulo - SP

Recebido em 27/6/01; aceito em 24/10/01

UTILIZATION OF REPEATABILITY AND REPRODUCIBILITY VALUES OBTAINED BY INTERLABORATORIAL PROGRAM AS PARAMETERS TO CONTROL PRECISION OF ANALYTICAL METHOD : WATER DETERMINATION BY KARL FISCHER METHOD. Values of repeatability ( $r$ ) and reproducibility (R) are parameters of an analytical method. They are related to the maximum difference between two results of analysis (permissible range) and can be obtained by interlaboratorial comparisons. They represent great importance for the quality control of the results and decisions about the fitness-for-purpose of the method.

Water determination in fuel oil samples were performed by 16 laboratories, using the Karl Fischer reaction, during the period from June 1997 to December 1999. Values of $r$ and $R$ were presented. Discussion is made in terms of the main factors that would be contribution to analytical method variabilities and uncertainties of final results.

Keywords: interlaboratorial program; analytical quality; Karl Fischer.

\section{INTRODUÇÃO}

A empresa produtora busca a melhor qualidade do seu produto final, a fim de se manter competitiva nos mercados atuais. Nesta busca, o laboratório analítico exerce papel fundamental, executando a análise de matérias-primas, produtos intermediários e finais. Decisões são tomadas baseadas nos resultados emitidos pelo laboratório, o que torna necessária a utilização dos sistemas de controle da qualidade analítica. Um dos meios empregados para esse controle é a participação em programas interlaboratoriais.

Comparações interlaboratoriais são usadas para determinar características de métodos, materiais ou laboratórios. Se o interesse for conhecer características de métodos, é necessário usar materiais adequados e laboratórios competentes para realizar as medições propostas. Se o problema é atribuir valores para certificar materiais de referência, o estudo deverá ser apoiado por laboratórios confiáveis de mais alta qualidade metrológica. Por outro lado, não é certo avaliar o desempenho de laboratórios a menos que sejam disponíveis amostras homogêneas a serem distribuídas para laboratórios utilizando-se de métodos validados.

Porém o benefício mais importante a ser usufruído por laboratórios que participam de comparações interlaboratoriais é ter meios de se comparar com laboratórios similares e identificar problemas que os laboratórios, quando trabalhando isolados, não poderiam descobrir. Dentre esses problemas podem ser citados: instruções descritas em normas não completamente seguidas, operadores com treinamento insuficiente ou inadequados, deterioração das práticas metrológicas inicialmente implantadas, utilização de reagentes fora de especificação

* e-mail: queenie@saofrancisco.edu.br

\# consultor em confiabilidade metrológica ou mesmo problemas de calibração de instrumentos empregados. Ainda existe o acesso a resultados obtidos por métodos diferentes, facilitando a comparação de desempenho de métodos em materiais idênticos e possibilitando verificar a influência de diversos fatores responsáveis pela variabilidade em resultados de análises.

Frente a todos esses argumentos, na prática, observa-se que os laboratórios que participam de comparações interlaboratoriais aproveitam muito pouco dos seus benefícios. Talvez por falta de esclarecimento quanto a essas possibilidades.

\section{PROGRAMAS INTERLABORATORIAIS}

A definição dada pela ISO REMCO (Reference Materials Committee) para Programa Interlaboratorial é bastante abrangente: "uma série de medições de uma ou mais propriedades, realizadas independentemente, por um grupo de laboratórios, em amostras de um material".

Por meio de um programa interlaboratorial específico, é possível a determinação de parâmetros de precisão para métodos de ensaios $^{1}$. Muitas normas brasileiras ainda não incluem informações a respeito desses parâmetros. Resultados de ensaios, mesmo obtidos com métodos normalizados, apresentam variabilidades. Na interpretação de resultados de ensaios, estas variabilidades devem ser consideradas.

Dois parâmetros de precisão, os denominados índices de repetibilidade - repê (r) e reprodutibilidade - Reprô (R), têm demonstrado ser necessários e suficientes para descrever as variabilidades de um método de ensaio ${ }^{2-4}$.

O índice $r$ indica a diferença máxima aceitável entre pelo menos dois resultados. Esses resultados referem-se a medidas obtidas em medições "quase iguais", dentro de um mesmo laboratório. Por sua vez, $\mathrm{R}$ indica a diferença máxima aceitável entre pelo menos dois 
resultados de laboratórios diferentes. O valor dos índices é determinado a partir da média dos erros internos de todos os laboratórios. Portanto só tem validade para uso dos laboratórios que participaram do programa interlaboratorial planejado para este fim.

Aplicados os testes de Cochran e de Grubbs ${ }^{1,5,6}$ para rejeição dos dispersos, as variabilidades dentre e entre laboratórios são calculadas através da análise pelas variâncias.

Os índices $\mathrm{r}$ e R são obtidos associando-se aos valores das variâncias (devido a repetições por laboratórios e a repetições entre os vários laboratórios) coeficientes que levam em consideração o grau de confiança desejado e o número de repetições a serem efetuadas pelo laboratório, na ocasião quando estes índices são empregados. Com um grau de confiança de $95 \%$, o coeficiente é igual a 2,77 válido para a diferença máxima permitida entre 2 resultados ${ }^{2}$.

A repê (r) e a Reprô ( $R$ ) devem ser mantidas sob controle, entendendo-se por "sob controle" a estabilidade, ao longo do tempo, para um determinado processo metrológico.

Os valores de r e R têm aplicação direta no dia a dia do laboratório após esse participar do programa interlaboratorial: para as questões internas do controle de produção em indústrias, para o controle da qualidade de resultados de ensaios em laboratórios e em situações de impasse, envolvendo a relação fornecedor-comprador, quando se tem de decidir sobre aceitação ou rejeição de bens e serviços.

Alguns casos são ilustrados abaixo quando, através de um programa interlaboratorial, foram obtidos $r=0,02$ e $R=0,03$.

\section{CASO 1:}

- laboratório obtém 2 repetições: $0,17 \%$ e $0,18 \%$

- a diferença entre as duas repetições de $0,01 \%$ é aceitável, pois $\mathrm{r}=0,02$; portanto os dois resultados são válidos.

\section{CASO 2:}

- laboratório obtém 2 repetições: $0,17 \%$ e $0,20 \%$

- a diferença entre as duas repetições de $0,03 \%$ é inaceitável $(\mathrm{r}=0,02)$; um dos valores deve ser rejeitado.

\section{CASO 3:}

- dois laboratórios: um laboratório obtém $0,18 \%$ e o outro $0,20 \%$ - a diferença entre os dois resultados de $0,02 \%$ é menor que o valor de $\mathrm{R}(0,03)$; portanto os dois valores são aceitos.

Neste contexto, pode-se dizer que programas interlaboratoriais representam esforço colaborativo com vistas ao domínio total de processos metrológicos. Os benefícios advindos dos esforços, que grupos de laboratórios se propõem a dedicar em conjunto, favorecem o trabalho para o estabelecimento de normas técnicas (métodos de ensaios e especificações), o aprimoramento da qualidade laboratorial e até a redução de custos de calibração de instrumentos, apontando a necessidade de ajustes e/ou manutenção.

\section{OBJETIVO}

Este trabalho tem o objetivo de divulgar a aplicação dos índices de repetibilidade e reprodutibilidade obtidos de programas interlaboratoriais planejados para este fim.

O programa interlaboratorial para determinação de água pelo método de Karl Fischer é apresentado nesse trabalho.

\section{EXPERIMENTO PARA A OBTENÇÃO DE ÍNDICES r E R}

Programas Interlaboratoriais para determinação de água pelo método de Karl Fischer, tendo como referência a norma ASTM D 1744-92 ${ }^{7}$, no período de 06/97 a 12/99, foram conduzidos sob a coordenação do Laboratório de Combustíveis e Lubrificantes da Divisão de Química do IPT para obtenção dos índices r e R.

Para a preparação das amostras, o laboratório homogeneizou, para cada rodada, 20 litros de óleo mineral básico sem aditivos proveniente de empresa de refinaria de óleos. A homogeneização foi feita em balde de polietileno sob agitação constante (agitador mecânico) por $30 \mathrm{mi}$ nutos. Um volume de aproximadamente $250 \mathrm{~mL}$ foi envazado em frascos de polietileno de alta densidade e hermeticamente fechados com tampa contendo batoque, evitando vazamentos. Os frascos foram protegidos por sacos de plásticos, selados e colocados em caixas de madeira. As amostras de óleo assim embaladas foram distribuídas aos 16 laboratórios participantes do programa colaborativo. As empresas sediadas no Estado de São Paulo retiraram suas amostras no IPT, enquanto que para aquelas sediadas fora de São Paulo, as amostras foram enviadas através de transportadoras especialmente contratadas.

Duas amostras de óleo foram distribuídas em cada ocasião para cada laboratório. O conjunto de 16 laboratórios foi mantido durante este período.

Estudos anteriormente conduzidos pelo laboratório indicaram ser possível garantir a estabilidade dos óleos minerais pelo prazo de 24 meses. No entanto, recomendações foram feitas aos laboratórios participantes que ensaiassem as duas amostras recebidas dentro do prazo máximo de uma semana. Todos os laboratórios adotaram os procedimentos seguindo a norma ASTM 1744-92 ${ }^{7}$, realizando duas repetições para cada amostra. As repetições, de preferência, deveriam ser em ocasiões diferentes, porém foi permitido que os laboratórios procedessem conforme sua rotina no trato das repetições em amostras.

\section{RESULTADOS}

Os resultados analíticos enviados pelos laboratórios foram avaliados quanto a valores dispersos, usando os critérios estatísticos segundo testes de Cochran, de Dixon e de Grubbs ${ }^{1,5,6}$.

Após essa etapa, os cálculos para valores de repê e Reprô foram efetuados usando a análise pela variância, considerando o grau de confiança de $95 \%$.

A Tabela 1 mostra os valores de repê (r) e Reprô (R) calculados a partir dos dados obtidos em cada ocasião (mês/ano indicado). A coluna que indica concentração representa os valores das médias de duas repetições obtidas pelos laboratórios participantes do programa.

É necessário verificar a existência de uma correlação entre a concentração e os índices de repê (r) e de Reprô (R). Se existir, então estes índices são dados pela função que representa a correlação existente, caso contrário estes índices são representados como constantes pelas respectivas médias ${ }^{1}$.

Tabela 1. Resultados de repê (r) e Reprô (R). Determinação de \% de água. Método Karl Fischer / ASTM D 1744 - 92

\begin{tabular}{lcccc}
\hline mês/ano & Am. & conc $^{*}(\%)$ & repê (r) $(\%)$ & Reprô $(\mathrm{R})(\%)$ \\
\hline $06 / 97$ & 1 & 0,018 & 0,003 & 0,017 \\
& 2 & 0,017 & 0,002 & 0,013 \\
$09 / 97$ & 1 & 0,019 & 0,002 & 0,023 \\
& 2 & 0,020 & 0,003 & 0,028 \\
$11 / 97$ & 1 & 0,024 & 0,008 & 0,029 \\
& 2 & 0,024 & 0,009 & 0,021 \\
$11 / 98$ & 1 & 0,024 & 0,002 & 0,022 \\
& 2 & 0,023 & 0,004 & 0,022 \\
$05 / 99$ & 1 & 0,030 & 0,005 & 0,043 \\
& 2 & 0,029 & 0,002 & 0,031 \\
$12 / 99$ & 1 & 0,026 & 0,009 & 0,022 \\
& 2 & 0,025 & 0,014 & 0,024 \\
\hline
\end{tabular}

* média de duas repetições 
A Figura 1 mostra os valores obtidos para os índices $\mathrm{r}$ e $\mathrm{R}$ no período que abrangeu as rodadas do programa (de 06/97 a 12/99) em função do nível da propriedade estudada (\% de água em amostras de óleo). A linha tracejada mais fina deveria representar a correlação entre a repê (r) e a concentração; porém não existe correlação (o coeficiente de correlação obtido, $\mathrm{R}^{2}=0,1212$, é muito baixo). Então a repê (r), para concentrações entre $0,015 \%$ e $0,035 \%$, pode ser representada pela linha contínua mais fina, considerando-se $\mathrm{r}$ como constante e igual a $0,005 \%$. A linha tracejada mais espessa deveria representar a correlação entre a Reprô $(\mathrm{R})$ e a concentração; porém não existe correlação (o coeficiente de correlação obtido, $R^{2}=0,5692$, é baixo). A Reprô (R), para concentrações entre $0,015 \%$ e $0,035 \%$, pode ser representada pela linha contínua mais espessa, considerando-se $\mathrm{R}$ como constante e igual a $0,025 \%$.

Temos portanto, repê $(r)=0,005 \%$ e Reprô $(R)=0,025 \%$.

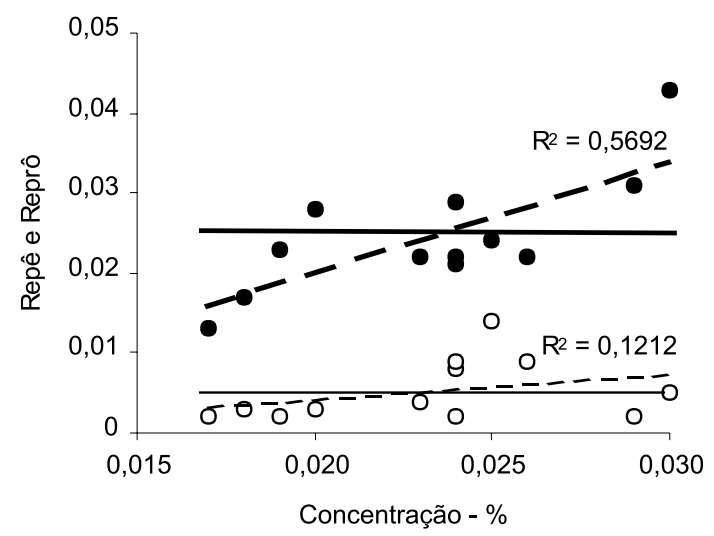

Figura 1. Índices $r$ e $R$ plotados em função da concentração Pontos vazados representam a repê $(r)$ e pontos cheios representam a Reprô $(R)$

\section{CONSIDERAÇÕES FINAIS}

Para níveis de concentração da ordem de $0,02 \%$, teríamos, segundo Horwitz ${ }^{8,9}$, variabilidade aceitável da ordem de $0,0015 \%$ e portanto, uma Reprô aceitável de $0,005 \%$, ou seja cinco vezes menos a que está sendo obtida no programa interlaboratorial.

Observa-se que os laboratórios individualmente apresentam boa precisão, porém permanecem erros sistemáticos entre laboratórios, demonstrados pelos altos valores obtidos para $\mathrm{R}$.

Os erros sistemáticos podem ser atribuídos a problemas no emprego da norma, envolvendo procedimentos operacionais que devem estar divergindo de um laboratório a outro. Muitos fatores podem estar contribuindo para isso, devendo ser identificados os problemas.

$\mathrm{O}$ estudo para diminuir as variabilidades possíveis entre laboratórios deve passar pela análise das etapas críticas do método da norma ASTM, entre as quais a padronização de solventes, o ponto final da reação e a sensibilidade dos instrumentos utilizados, considerando-os como os fatores principais.

O reagente convencional de Karl Fischer utilizado na titulação de água consiste de uma solução contendo iodo, dióxido de enxofre e uma base (piridina ou imidazol ou outra) em solvente na maioria das vezes utilizado o metanol ${ }^{10}$. Solventes são usados não apenas como parte do reagente de Karl Fischer, mas também como meio titulante, sendo que têm influência sobre a estequiometria da reação e correspondente alteração no título do reagente. A razão estequiométrica entre iodo e água na reação de Karl Fischer é, em geral, de $1: 1$ a $1: 2^{10}$.

A reação de Karl Fischer é de $1^{\mathrm{a}}$ ordem com respeito a $\mathrm{I}_{2}, \mathrm{SO}_{2} \mathrm{e}$ $\mathrm{H}_{2} 0$, porém somente em casos quando o valor de $\mathrm{pH}$ do meio de trabalho corresponde ao $\mathrm{pH}$ do reagente de Karl Fischer, ou seja, $\mathrm{pH}$ 5,5 a 8 com bases como piridina ou imidazol ${ }^{11,12}$.

A velocidade da reação de Karl Fischer é influenciada pela presença ou não de algumas amidas (ex: formamida ou $\mathrm{N}$-metil formamida) na mistura reagente com metanol ${ }^{13}$, sendo que sua presença favorece a ocorrência de reações secundárias, dificultando a deteção do ponto final da titulação. Enfim, fatores que atuam nas condições de cinética e estequiometria da reação representam fontes de incerteza na determinação de água pelo método de Karl Fischer.

Outras fontes de variabilidades que contribuem na incerteza do resultado nesta determinação têm a ver com os componentes da matriz da amostra em estudo, outros que não a própria água, que reagem com o titulante. Mas este tipo de problema seria comum a todos os laboratórios participantes do programa interlaboratorial. O que pode ser apontado como item de investigação seria a influência da massa da porção inicial da amostra utilizada pelos vários laboratórios, bem como a resposta na determinação do "branco"; apesar de que é sabido que a determinação da massa inicial é obtida com incerteza bem menor comparada às incertezas obtidas com volumes envolvidos em diluições e na detecção do ponto de viragem na titulação $0^{14}$.

Aplicadas as ações corretivas necessárias, outras amostras deverão ser analisadas por meio de nova rodada de programa; valores para precisão deverão ser avaliados para o acompanhamento da evolução dos trabalhos, até a obtenção de índices menores e estáveis ao longo do tempo.

Os índices r e R são dinâmicos e requerem a sua revisão, ou seja, a repetição de rodadas do programa a intervalos periódicos. Apesar de não garantirem a exatidão ${ }^{15,16}$, o seu emprego é muito útil no controle de produção, no controle da qualidade de resultados analíticos em medições, na relação fornecedor-recebedor e, enfim, na validação de resultados.

Deve ser salientado que é indispensável um programa interlaboratorial para obtenção dos índices $r$ e R. É desejável associar índices a tipos de equipamentos, repetições e metodologias aplicadas às medições.

A utilização de valores de $\mathrm{r}$ (repê) e R (Reprô) contribui para o monitoramento da precisão de metodologias, representando subsídios para a implementação de programas para a melhoria da qualidade em laboratórios de medições.

\section{REFERÊNCIAS}

1. International Standard Organization, ISO 5725-1990; Accuracy (Trueness and Precision) of Measurement Methods and Results, Part 1 - General Principles and Definitions, Suíça.

2. Kuselman, I.; J. Qual.,Compar. Reliab. in Chem. Meas. 1999, 4, 511.

3. Waeny, J.C.C; Publicação IPT 1156, 1985.

4. Waeny, J.C.C; ABCQ Nota Técnica 17/90; Repetitividade e Reprodutividade III, 1990, São Paulo: SP.

5. Grubbs, F.E.; Technometrics 1969, 11, 1.

6. Taylor, J.K.; Quality Assurance of Chemical Measurements, Lewis Publishers, Inc., CRC Press: USA, 1987 (ISBN - O - 87371-097-5), p. 36.

7. American Society for Testing \& Materials, ASTM Standard D 1744-92; Standard Test Method for Determination of Water in Liquid Petroleum Products by Karl Fischer Reagent, Philadelphia: Pa.

8. Horwitz, W.; Kamps, L.R.; Boyer, K.W.; J. AOAC Int. 1980, 63, 1344.

9. Thompson, M.; Analyst 2000, 125, 385.

10. Kuselman, I.; Sherman, F.; J. Qual., Compar. Reliab. in Chem. Meas. 1999, 4, 230.

11. Cedergren, A.; Anal.Chem.1996, 68, 784.

12. Verhoof, J.C and Barendrecht, E.; J. Eletroanal.Chem. 1976, 71, 305.

13. Cedergren, A.; Talanta 1978, 25, 229.

14. EURACHEM/CITAC Guide; Quantifying Uncertainty in Analytical Measurement, $2^{\text {nd }}$ ed., Helsinki Workshop, 1999.

15. De Brièvre, P.; J. Qual.,Compar. Reliab. in Chem. Meas. 1999, 4, 342.

16. De Brièvre, P.; J. Qual.,Compar. Reliab. in Chem. Meas. 1999, 4, 387. 